\title{
Apego Adulto y Mentalización en Hombres que Han Ejercido Violencia Hacia su Pareja
}

\section{Adult Attachment and Mentalization in Men Who Have Used Violence Toward Their Partner}

\author{
Valezka Pimentel y María Pía Santelices \\ Pontificia Universidad Católica de Chile
}

\begin{abstract}
El objetivo de este estudio fue evaluar el apego adulto y la mentalización en 20 hombres condenados por violencia hacia su pareja, en comparación con 20 hombres sin antecedentes de violencia hacia su pareja, de entre 30 y 50 años de edad. Ambos grupos fueron reclutados intencionadamente de un hospital público de la Región de Valparaíso, Chile. Se utilizó el Conflict Tactics Scales-2 (CTS-2) de Straus, Hamby, Boney-McCoy y Sugarman (1996) para distinguir niveles de violencia hacia la pareja, la Escala de Experiencia en Relaciones Cercanas (ERC) de Brennan, Clark y Shaver (1998) para evaluar apego adulto y el test Reading the Mind in the Eyes de Baron-Cohen, Wheelwright, Hill, Raste y Plumb (2001) para evaluar mentalización. Se realizaron pruebas de $\chi^{2}$, test exacto de Fisher y $U$ de MannWhitney para las variables de apego y mentalización. Los resultados apuntan a que hombres que ejercen violencia presentarían más apego inseguro y mayor ansiedad en el apego hacia sus parejas que los que no tienen antecedentes de violencia, resultando los puntajes en los niveles de mentalización similares en ambos grupos.
\end{abstract}

Palabras clave: violencia hacia la pareja, apego adulto, ansiedad, evitación, mentalización

\begin{abstract}
The objective of this study was to assess adult attachment and mentalization of 20 men convicted for violence against their partners compared with 20 men with no history regarding this kind of violence, whose ages ranged from 30 to 50 years old. Both groups were purposively recruited from a public hospital in the Region of Valparaíso, Chile. The Conflict Tactics Scales-2 (CTS-2) by Straus, Hamby, Boney-McCoy, and Sugarman (1996) was used to distinguish levels of violence against partners, the Experience in Close Relationships Scale (ECR) by Brennan, Clark, and Shaver (1998) was employed to assess adult attachment, and the Reading the Mind in the Eyes test by Baron-Cohen, Wheelwright, Hill, Raste, and Plumb (2001) was used to assess mentalization. $\chi^{2}$, Fisher's exact test, and the MannWhitney $U$ test were used to analyze the attachment and mentalization variables. Results indicate that men who exert violence display more insecure attachment and greater anxiety regarding their attachment to their partners than those with no history of violence, with similar mentalization levels being observed in both groups.
\end{abstract}

Keywords: partner violence, adult attachment, anxiety, avoidance, mentalization

Las cifras recientes de la prevalencia mundial indican que el 35\% de las mujeres del mundo ha sufrido violencia por parte de su pareja (Organización Mundial de la Salud [OMS], 2016). En Chile, en la encuesta nacional de victimización por violencia intrafamiliar y delitos sexuales (Servicio Nacional de la Mujer, 2011) se observa que un $31,9 \%$ de las mujeres entre 15 y 65 años declara haber sufrido alguna forma de violencia y el 74\% de ellas indica que la recibió de parte de su pareja o ex pareja. El maltrato de la pareja es especialmente problemático, porque tiene lugar en el ámbito familiar privado, lo que lo convierte en un campo difícil para la intervención y la ayuda (Organización Panamericana de la Salud, 2002).

El abordaje fundamental para estudiar el tema de la violencia hacia la pareja ha sido desde el contexto sociocultural, donde la comprensión, en términos amplios, es que la violencia se explica a partir de la legitimación de la socialización masculina del ejercicio de la violencia hacia las mujeres (American Psychological Association, 1996; Blázquez Alonso, Moreno Manso \& García-Baamonde Sánchez, 2010; Bonino, 2008; Echeburúa, Amor \& de Corral, 2009; Ibaceta Watson, 2004; Johnson \& Ferraro, 2000), explicación que se ha ido ampliando en la actualidad hacia las relaciones interpersonales (Hamel, 2009).

Valezka Pimentel Recabal y María Pía Santelices Álvarez, Escuela de Postgrado de Psicología, Pontificia Universidad Católica de Chile, Santiago, Chile. Chile.

El artículo es parte de la tesis para optar al Grado de Magíster en Psicología Clínica de la Pontificia Universidad Católica de

La correspondencia relativa a este artículo debe ser dirigida a Valezka Pimentel Recabal, Unidad de Salud Mental, Hospital de Quilpué, Thompson 1245, Quilpué, Chile. E-mail: valezkap@gmail.com 
PIMENTEL Y SANTELICES

El modelo planteado por Bowlby $(1969 / 1976,1980 / 1984,1988)$ propone que la relación con la figura de apego desarrolla un patrón de vinculación que se expresa en las relaciones adultas y que puede ser, en términos generales, un apego seguro o inseguro, de acuerdo a la calidad de la relación establecida entre el adulto y el bebé. En términos evolutivos, este patrón se desarrolla a partir de las conductas que establecen los infantes, y posteriormente los adultos, para lograr la proximidad de las figuras de apego en momentos de temor, ansiedad o estrés, lo que le confiere a estas conductas un estatus de sobrevivencia y auto-conservación. Estas conductas que permiten establecer relaciones significativas entre los cuidadores y los niños y entre los adultos surgen a partir de representaciones mentales internalizadas, a las cuales Bowlby (1969/1976, 1980/1984) definió como pautas o estilos de apego. En el caso de los adultos estos reciben el nombre de modelos internos o mapas representacionales de las relaciones (MOI). Estos modelos pueden ser definidos como representaciones o esquemas que tiene un individuo de sí mismo y los otros (Marrone, 2001), que guían la manera en que se funciona en diversos contextos interpersonales, especialmente aquellos que propician la intimidad.

La imagen de sí mismo está relacionada con el grado en que se experimenta ansiedad acerca de ser rechazado o abandonado, de modo tal que las personas que poseen una visión positiva de sí mismas tenderían a experimentar baja ansiedad respecto de esta posibilidad, dado que se consideran dignas de ser amadas y cuidadas. Por el contrario, aquellas que poseen una visión negativa de sí mismas tenderían a manifestar preocupación y temor frente al abandono de la figura de apego.

Por su parte, la imagen del otro estaría asociada al grado de evitación que la persona manifiesta respecto de las relaciones cercanas. Aquellas que tienen una imagen positiva del otro en términos de confiabilidad y disponibilidad tendrán mayor facilidad para establecer relaciones cercanas con otro. Por el contrario, las que tienen una visión negativa de los demás, como poco receptivos, tenderán a evitar involucrarse más íntimamente en los vínculos (Guzmán \& Contreras, 2012).

Los MOI antes descritos son explícitamente relacionales, es decir, incluyen imágenes de sí y de los otros en situaciones altamente interdependientes, más que en aislamiento (Shaver, Collins \& Clark, 1996). Además, pueden ser usados para predecir el comportamiento en diversos ámbitos del funcionamiento humano, pues sirven como filtros a través de los cuales se interpretan las relaciones con los otros. Ambas dimensiones se articulan en patrones sistemáticos de expectativas, necesidades, emociones, estrategias de regulación emocional y conducta social, denominados estilos de apego (Feeney \& Noller, 1996/2001), que influyen en la manera en la que un individuo se involucra en relaciones cercanas.

Siguiendo los planteamientos de Bowlby respecto de la representación de sí mismo y de los otros, Bartholomew y Horowitz (1991) desarrollaron un modelo de cuatro categorías de apego, a las cuales subyacen dos dimensiones, la ansiedad del abandono y la evitación de la cercanía emocional: (a) seguro, que aúna una idea positiva de sí mismo y de los demás, evidenciando, por tanto, baja ansiedad y evitación ante los contactos interpersonales de mayor intimidad; (b) desentendido o evitativo, con una idea positiva de sí mismo y negativa de los demás y, por lo tanto, manifiesta baja ansiedad y alta evitación; (c) preocupado, con una idea negativa de sí y positiva de los demás, acompañada de alta ansiedad y baja evitación y (d) temeroso, con una idea negativa tanto de sí como de los otros, asociado a alta ansiedad y alta evitación.

Dado que los estilos de apego se expresan a lo largo de la vida en nuevas relaciones, es comprensible que jueguen un rol importante en las relaciones de pareja, por ser vínculos que favorecen la intimidad y el cuidado mutuo (Fraley \& Shaver, 2000). Hazan y Shaver (1987), quienes desarrollaron las primeras investigaciones en este ámbito, propusieron que las relaciones de pareja pueden ser conceptualizadas como relaciones de apego, las que se encontrarían influidas, en parte, por las experiencias con los cuidadores, internalizadas en los MOI.

De acuerdo a estos autores, existiría un paralelo entre el apego del infante y su cuidador y el apego en las relaciones de pareja, expresado en el deseo de mantener la proximidad física, la confianza en la figura de apego para el confort y la visión de esta como una fuente de seguridad en momentos de estrés. Sin embargo, el apego adulto difiere del apego infantil en diversos aspectos: en las relaciones de pareja la naturaleza de la relación es recíproca, las necesidades afectivas previamente satisfechas por los cuidadores se transfieren gradualmente hacia las parejas significativas en la adultez (Fraley \& Shaver, 2000) y el apego adulto se manifiesta en la calidad de las relaciones y en la regulación del afecto ante situaciones de amenaza o conflicto (Feeney \& Noller, 1996/2001; Hazan \& Shaver, 1987).

Los MOI, entonces, dirigen las respuestas afectivas y conductuales con las que los adultos responden a los requerimientos de los niños a su cargo y fueron formados, en gran parte, durante la propia infancia de 
estos. Esto quiere decir que, de acuerdo al modo como estos adultos fueron tratados por sus propios cuidadores cuando niños, se han desarrollado sus propios MOI. De esta manera, la figura de apego refleja su experiencia interna y busca producir una respuesta emocional apropiada en el niño, de modo de regular afectivamente la conducta propia y del otro, proceso que no es consciente y expresa en gran medida la forma de apego de la persona que está a cargo, sea su madre o cuidador/a. Este modo de regulación afectiva de la experiencia subjetiva es llamada por Fonagy y Target (1997) reflexiva (reflective, mirroring), conformando un constructo mayor llamado mentalización.

Tanto el apego como la mentalización se han incorporado en los desarrollos teóricos y/o empíricos en relación a la violencia. El caso del apego en el adulto que agrede a sus parejas ha sido ampliamente abordado (Babcock, Jacobson, Gottman \& Yerington, 2000; Dutton \& Golant, 1995/1997; Holtzworth-Munroe, Meehan, Herron, Rehman \& Stuart, 2000; Lawson \& Malnar, 2011; Lyons-Ruth, Alpern \& Repacholi, 1993; LyonsRuth, Easterbrooks \& Cibelli, 1997; Main \& Solomon, 1990; Waltz, Babcock, Jacobson \& Gottman, 2000). Sin embargo, aún hay controversia en relación al tipo de apego que predomina en esta población, específicamente en hombres que no han sido recluidos por este tipo de violencia, ya que la mayoría de los estudios se centra en población penitenciaria. En el caso de la mentalización, su abordaje ha sido más bien en el contexto clínico (Fonagy, 2003; Fonagy \& Bateman, 2006; Fonagy, Target \& Gergely, 2000), con bajo nivel de evidencia empírica, especialmente en el caso de hombres que agreden a sus parejas.

Por lo tanto, el objetivo de este estudio fue detectar si existen diferencias en el apego adulto y la mentalización en hombres que han sido condenados por ejercer violencia hacia sus parejas, en comparación con aquellos que no tienen antecedentes de violencia hacia sus parejas. Las preguntas que guiaron esta investigación fueron: ¿Existen diferencias en el apego adulto y la mentalización en hombres que ejercen violencia hacia sus parejas de aquellos que no tienen antecedentes de violencia hacia sus parejas? y ¿cómo estos aspectos pueden aportar a la comprensión de la violencia hacia la pareja?

\section{Apego y Violencia}

Desde la perspectiva del apego, se introduce una mirada etológica y relacional en la constitución de los vínculos tempranos, en la medida que la relación con la figura de apego desarrolla un modo de vinculación que se expresa en las relaciones adultas, permaneciendo relativamente estable. En esta línea, Bowlby (1980/1984) plantea que en el desarrollo del ser humano hay un proceso de exclusión defensiva de toda la información que produce dolor, con lo cual se originan exacerbaciones o desviaciones en el desarrollo del apego, que opera de manera automática en las relaciones afectivas adultas, influyendo en la visión de sí mismo y la de los otros.

El apego no explica la conducta, pero sí ofrece un método de análisis sobre los procesos psicológicos que median en la agresión, tales como ciertos déficits cognitivos o emocionales (falta de empatía o tendencia a la impulsividad) (Ansbro, 2008). Loinaz, Echeburúa y Ullate (2012) plantean que la evaluación del apego forma parte de los protocolos que se utilizan con los hombres violentos con la pareja, para diferenciar subtipos de agresores (Amor, Echeburúa \& Loinaz, 2009; Babcock et al., 2000; Dutton, 2008; Holtzworth-Munroe et al., 2000; Holtzworth-Munroe \& Stuart, 1994) o para evaluar resultados terapéuticos (Lawson, Barnes, Madkins \& Francois-Lamonte, 2006). Sin embargo, la mayoría de los estudios se ha centrado en hombres en reclusión carcelaria y orientados a la tipificación de los agresores (Dutton, 2008, 2011; Echeburúa et al., 2009; Feeney, 1999; Jacobson \& Gottman, 1998/2001; Loinaz et al., 2012).

Desde esta perspectiva, Dutton $(2008,2011)$ propone que la agresión en la pareja puede entenderse como un comportamiento de protesta dirigido a la figura de apego, precipitado por amenazas de separación o abandono. De acuerdo a este autor, los hombres maltratadores mostrarían más probabilidades de depender de una relación y de sentir ansiedad tanto ante la separación como a la cercanía, que es la base del apego ansioso. Por ello, es probable que busquen parejas sobre las cuales puedan ejercer un control a través del cual manejen la experiencia infantil de un acercamiento fallido (Dutton, 2011).

Por su parte, Fonagy et al. (2000), desde una perspectiva clínica de hombres que han ejercido violencia, plantean que el apego ansioso puede estar relacionado con la violencia, pero no puede proporcionar una explicación suficiente, ya que, por una parte, el apego ansioso es demasiado común en la población y, por otra, este sería una estrategia interpersonal relativamente estable en la edad adulta. Señala que ninguna de las estrategias relacionales que establece el apego inseguro se asemeja al comportamiento incontrolado de los hombres violentos. 
La violencia en general ha sido mayoritariamente relacionada con el apego desorganizado (Lyons-Ruth, et al., 1993, 1997; Main \& Solomon, 1990; Moss, Parent, Gosselin, Rousseau \& St-Laurent, 1996), caracterizado por una actuación parental estresante e incoherente para el niño, como maltrato infantil, abuso, negligencias o la presencia de patologías en los cuidadores. Estas dinámicas propician la generación de modelos mentales en conflicto, puesto que la figura de apego es fuente de protección y de amenaza a la vez (Main \& Solomon, 1990), y conllevarían al desarrollo de ciertas dificultades importantes en la adultez, como sentido inestable del sí mismo, irritabilidad, inestabilidad emocional e impulsividad, entre otras (Fonagy et al., 2000). Walker (1979) propone que la evidencia clínica sugiere que estos hombres, lejos de desestimar la importancia de las relaciones, a veces van a los extremos para mantener las relaciones disfuncionales con sus víctimas después de episodios violentos, expresando arrepentimiento, desesperación y un compromiso con el cambio; esta actitud no sería característica de un estilo de apego inseguro, aunque sí podría relacionarse más bien con el apego desorganizado. En sus estudios con niños vulnerables, Lyons-Ruth et al. (1993) proponen que las observaciones de seguimiento muestran una asociación entre el apego desorganizado y los niveles anormales de comportamiento agresivo. Postulan, además, que este desarrollo en la edad adulta se manifiesta como una necesidad imperiosa de controlar al otro, tendiendo a establecer relaciones en las que la pareja actuaría como un vehículo para los estados del sí mismo que le son intolerables. En su desarrollo teórico, Renn (San Miguel, 2006) propone que el ejercicio de la violencia por parte del hombre partiría de un trauma infantil que incluiría la separación, abandono, abuso y/o negligencia que se producen en el contexto de un sistema de apego desorganizado. Este tipo de apego con la figura primaria, sumado a experiencias traumáticas y un sistema de representación caracterizado por la disociación, da lugar a la distancia emocional e incapacidad para regular los estados afectivos. El efecto de todo lo anterior sería una percepción distorsionada de la pareja y una conducta controladora indebida. Ante un abandono percibido o real, se activaría el miedo y el sistema de apego desorganizado, lo que trae aparejado desregulación afectiva, retraumatización y conducta violenta.

La investigación en general ha encontrado que los agresores de pareja, en comparación con sujetos no violentos, muestran apego inseguro, poca estabilidad emocional y gran ansiedad frente al rechazo o abandono por parte de la pareja (Babcock et al., 2000; Dutton, 2008; Holtzworth-Munroe, Meehan, Herron, Rehman \& Stuart., 2003). Asimismo, el apego inseguro con la pareja es más frecuente en hombres que han ejercido violencia hacia ella que aquellos que no lo han hecho (Holtzworth-Munroe et al., 2003; Lawson \& Malnar, 2011; Waltz et al., 2000).

Doumas, Pearson, Elgin y McKinley (2008) evaluaron el estilo de apego hacia la pareja en 70 varones, concluyendo que hombres con apego evitativo y mujeres con apego ansioso serían predictores de violencia. Asimismo, Babcock et al. (2000) evalúan (con la entrevista de apego adulto, AAI) estilos de apego en 23 hombres violentos y 13 no violentos hacia sus pareja, concluyendo que los primeros muestran más inseguridad en el apego que los últimos. El 23\% de los sujetos agresores de este estudio se mostró con apego seguro. Por otro lado, los estilos de apego inseguro, que presentan alta ansiedad (preocupado y temeroso), se han relacionado con la agresión a la pareja en numerosos estudios (Lawson \& Brossart, 2009; Mauricio \& López, 2009; Mikulincer, Shaver \& Pereg, 2003). La asociación entre estilos de apego inseguro y ciertas variables como una menor autoestima, mayor impulsividad o ira también ha sido encontrada en una muestra española de agresores de pareja (Loinaz, Echeburúa \& Torrubia, 2010). Por su parte, en un estudio en los Países Bajos se comparan 23 hombres agresores con apego seguro con un grupo de maltratadores con apego inseguro (Buck, Leenaars, Emmelkamp \& van Marle, 2014). Los resultados muestran que la asociación entre el apego inseguro y la violencia doméstica puede ser explicada por la ansiedad de separación y desconfianza en la pareja. Por otra parte, solo la desconfianza hacia la pareja aumentaba el riesgo de maltrato por sobre el apego inseguro.

Por su parte, O'Leary y Cascardi (1998) proponen que los hombres que ejercen violencia hacia sus parejas no tienen patrones de apego distintos al de otros hombres. Plantean que es probable que haya muchos caminos diferentes para llegar a ser violento con la pareja y que el apego inseguro y la baja regulación afectiva es solo uno de ellos. En Chile, Barría Muñoz (2013) señala, respecto de una muestra de 732 hombres que han ejercido violencia hacia sus parejas, que solo el $21,7 \%$ de hombres agresores presenta apego seguro, mientras que un $66,7 \%$ de hombres que no han ejercido violencia presenta este tipo de apego. Asimismo, propone que en los hombres que ejercen violencia predomina el apego ansioso con un $32 \%$ y el apego temerosodesorganizado con un 34\%. Pese a esta diferencia en ambos grupos, Barría Muñoz y Macchiavello Rodríguez (2012) advierten que los estilos de apego "serían una variable moderadora que podría influir en los procesos de expresión funcional o disfuncional de la ira, y en la regulación emocional del sujeto" (p. 62). 
Si bien la evidencia empírica aún no ha confirmado el modo preciso en que se relacionaría el apego de los hombres con el ejercicio de la violencia, existe acuerdo en que ambas variables, apego y violencia, se encontrarían relacionadas.

Un factor importante para comprender esta relación es que, al explicar la violencia en la pareja con la teoría de apego, no solo se alude a la idea de que es en la relación temprana con la figura de apego en la que se asientan las bases para las relaciones futuras (patrón de relación), sino que también se instala un modo de pensar y un modo de representarse a sí mismo y al otro en una relación vincular. Así, quienes han tenido experiencias de violencia en sus etapas tempranas tenderán no solo a repetir el patrón de relación, en algunos casos, sino que también poseerán un modo de mentalizar las relaciones afectivas que retroalimentan las relaciones violentas y abusivas (Barudy, 1998).

\section{Mentalización o Función Reflexiva}

Para Fonagy, Target, Steele y Steele (1998) la mentalización o función reflexiva consiste en la capacidad de imaginar y entender los estados mentales en uno mismo y en otros; es la habilidad de dar una interpretación convincente a la conducta propia y de otros, a partir de los estados mentales subyacentes. Esta capacidad está intrínsecamente ligada al desarrollo del sí mismo, a su organización interna gradualmente elaborada y a su participación en la sociedad (Fonagy et al, 1998). Asimismo, permite diferenciar entre los procesos mentales y emocionales intrapersonales y los procesos afectivos interpersonales (Fonagy, 2003; Fonagy et al., 2000). Esta teoría pone énfasis en las experiencias sociales tempranas como un moderador esencial de las capacidades mentales. Cuando estas capacidades son fallidas o se alteran, son centrales para la consideración de los desórdenes psicológicos en la edad adulta. Por ejemplo, la incapacidad de visualizar la causalidad psicológica y psicosocial puede ser la consecuencia de la inhibición y/o de la malformación del desarrollo del proceso psicológico que soporta estas capacidades y que se mantendría relativamente estable a través del tiempo (Fonagy et al., 1998).

El desarrollo cognitivo sería, entonces, un producto de un complejo proceso psicológico que emerge en la proximidad a otro ser humano en la temprana infancia, pero la calidad del desarrollo de esta capacidad también dependerá de la calidad de esa temprana relación (Wimmer \& Perner, 1983). Por ende, en ausencia de vínculos afectivos seguros, en circunstancias en que las llamadas figuras de apego no logran ejercer tareas que estimulen la capacidad mentalizadora en los niños, como actividades de atención conjunta, o de mantener con el bebé un estado de sintonía emocional, los niños tendrán dificultad para discernir sus propios estados mentales o los estados mentales de los demás, o bien a no diferenciar los estados internos de los ajenos (Allen, 2006; Fonagy et al., 1998).

Fonagy (2003) afirma que para que se produzca la violencia debe darse una precondición esencial: la vulnerabilidad a una regresión al estado de equivalencia psíquica, en la que lo interno es igualado con lo externo. Para este autor, la violencia es normalmente provocada cuando una idea, un sentimiento, un prejuicio o una suposición se confunden con la realidad física. El acto de violencia refleja la errónea creencia de que un acto físico puede erradicar un estado mental. El empleo de la violencia es también entendido como resultado de una distorsión en el desarrollo afectivo y muestra un déficit, una falta de apropiación experiencial de las acciones (Fonagy \& Target, 2008).

En el contexto del trabajo terapéutico con hombres que han ejercido violencia hacia sus parejas, Jenkins (1990) plantea que estos tienen valores y creencias que actúan como limitantes para aceptar la responsabilidad por sus conductas agresivas y para desarrollar relaciones respetuosas con otros seres humanos. Estas limitantes se reflejan en las formas en que los hombres agresores construyen explicaciones de sus conductas y modelos de relación con los otros; asimismo, la conducta de los agresores tiende a ser bastante consistente con su visión restringida de ellos y sus relaciones. En este sentido, la función reflexiva de los hombres que ejercen violencia pudiese presentar características diferentes de los hombres que no han ejercido violencia hacia sus parejas. Por su parte, Fonagy (2003), explicando esta relación, afirma que "los actos violentos contra las mujeres son a menudo cometidos por hombres con insuficiencia en la capacidad de mentalización" (p. 4), a la vez que propone que dar una explicación lineal y entender la conducta violenta de los hombres como déficit en la mentalización sería demasiado simplista, ya que existe también un contexto relacional en el cual la violencia ocurre. Así, si bien teóricamente esta relación posee argumentación probable de ser establecida, aún no cuenta con suficiente desarrollo empírico que permita corroborarla. 
PIMENTEL Y SANTELICES

Dolan y Fullam (2004) realizan un estudio con 89 hombres con personalidad antisocial (ASPD) que han cometido crímenes violentos, divididos en psicópatas y no psicópatas, y 20 sujetos control. Ambos grupos completaron una batería de tareas ToM (teoría de la mente) sin diferencias entre los grupos. Cabe destacar que el grupo de hombres no psicopáticos mostraron alteraciones en el reconocimiento de emociones básicas, en comparación con el grupo control y los psicopáticos. Los autores concluyen que los déficits en la capacidad de mentalización en hombres con personalidad antisocial son sutiles. Para la mayoría de los criminales con ASPD las habilidades ToM están relativamente intactas y pueden tener una función adaptativa en el mantenimiento de un estilo de vida criminal. Los déficits principales se relacionan más con falta de preocupación por el impacto en las víctimas potenciales. Los hallazgos también sugieren que tentativamente ASPDs con rasgos neuróticos pueden tener más deterioro en la capacidad de mentalización que los psicópatas propiamente tales.

Gómez, Molina y Arana (2013) realizan una investigación con 20 hombres entre los 25 y 50 años recluidos en una institución carcelaria en Colombia, vinculados al conflicto armado y con historia delictiva, evaluando la mentalización con dos instrumentos, uno de los cuales fue el Reading the Mind in the Eyes (Baron-Cohen, Jolliffe, Mortimore \& Robertson, 1997). La comparación se realizó con las puntuaciones normativas en sujetos sin antecedentes de comportamiento violento. El grupo vinculado al conflicto armado presentó una media inferior al grupo normativo, concluyendo que existen mayores dificultades en el reconocimiento y procesamiento emocional en esa población.

Capaldi y Kim (2007) proponen que los hallazgos de los estudios de desarrollo en violencia hacia sus parejas por parte de los hombres han indicado que se necesitan modelos que sean más abiertos a indagar en explicaciones tanto intrapersonales como interpersonales y que el modelo dinámico de desarrollo de la violencia de pareja puede ser desarrollado para reflejar esta necesidad.

En esta línea, los aspectos que se investigaron en este estudio, el apego adulto y la mentalización, permiten ampliar el foco de estudio de la violencia por parte de los hombres hacia variables intersubjetivas, que puedan orientar hacia una arista diferente en la comprensión del fenómeno de la violencia en pareja. Las hipótesis que guiaron esta investigación fueron: la primera es que existen diferencias en relación al estilo de apego entre los hombres que presentan antecedentes de violencia en la pareja de los que no la han ejercido. La segunda se relaciona con que los hombres que han ejercido violencia hacia su pareja presentan un menor nivel de mentalización que los hombres que no han ejercido violencia. Y, finalmente, que los hombres que han ejercido violencia hacia su pareja presentan un estilo de apego más inseguro que los hombres que no tienen antecedentes de aquello.

\section{Método}

\section{Diseño}

Este estudio se llevó a cabo a través de un diseño transversal, descriptivo, correlacional y no experimental. Se utilizó una metodología cuantitativa, contrastando la información obtenida entre dos grupos: un grupo de estudio de hombres condenados por haber ejercido violencia hacia su pareja y otro de comparación de hombres que no presentaban antecedentes de violencia hacia sus parejas.

\section{Participantes}

Los participantes, seleccionados a través de una muestra intencionada, fueron dos grupos de 20 hombres entre 30 y 50 años cada uno. El promedio de edad fue de 39 años $(D E=7,7)$. Un 47,5\% de los participantes reportó ser soltero, un $45 \%$, casado y un 7,5\%, separado. El 77,5\% de los participantes afirmó tener pareja actual. La gran mayoría de los participantes reportó tener hijos (80\%), con un promedio de 1,5 hijos $(D E=$ 1,1) y un rango entre 1 y 4 hijos.

Ambos grupos corresponden a usuarios del sistema público de salud, pertenecientes al tramo A o B del Fondo Nacional de Salud de Chile, es decir, correspondientes a personas indigentes, carentes de recursos o causantes de subsidio familiar (Ley 18.020 de 1981; Chile, Ministerio de Trabajo y Previsión Social, 2017), o bien personas que perciben un ingreso imponible mensual promedio entre $\$ 250.000$ y $\$ 360.000$ (entre 375 y 545 dólares).

El grupo de estudio estuvo compuesto por hombres derivados a un hospital público de la Región de Valparaíso, Chile, por los tribunales de justicia por el "delito de lesiones menos graves en el contexto de 
violencia intrafamiliar" a atención psicológica para el control de impulsos, como condición especial del artículo 9, letra d de la Ley 20.066 de 2005 (Chile, Ministerio de Justicia, 2016).

El grupo de comparación fue reclutado a través de la derivación de los médicos tratantes y/o enfermeras de los asistentes al consultorio de especialidades del mismo hospital.

La primera autora realizó entrevistas individuales o de dupla médico-enfermera de cada servicio clínico del hospital, entregando los objetivos del estudio, los criterios de derivación y los procedimientos.

\section{Instrumentos}

Entrevista sociodemográfica. Al inicio de la aplicación de la batería de test se realizaron algunas preguntas sobre información sociodemográfica de cada participante: edad, nivel educacional, estado civil y ocupación.

Cuestionario Autodiagnóstico de Riesgos en el Uso de Alcohol (Use Disorders Identification Test, AUDIT). El consumo de alcohol es un factor que gatilla la violencia hacia la pareja (Amor et al., 2009; Barría Muñoz, 2013), por lo cual ambos grupos fueron evaluados previamente con este instrumento a fin de controlar el efecto del uso/abuso de alcohol en los resultados. Fue desarrollado por la Organización Mundial de la Salud (OMS) como un método de tamizaje del consumo excesivo de alcohol y como un instrumento de apoyo en la intervención breve (Babor, Higgins-Biddle, Saunders \& Monteiro, 2001).

El AUDIT es un cuestionario de 10 ítems, separados en tres dominios, de acuerdo a la siguiente distribución: Dominio 1, sobre consumo de riesgo de alcohol, corresponde a los ítems 1 a 3; Dominio 2, sobre síntomas de dependencia, incluye los ítems 4 a 6 y Dominio 3, sobre consumo perjudicial de alcohol, incluye los ítems 7 a 10. Los ocho primeros ítems tienen cinco categorías de respuesta ( 0 a 4 puntos) y los dos últimos poseen tres alternativas cada uno (0, 2 y 4 puntos). En su conjunto, la escala del test tiene un rango entre 0 y 40 puntos.

De acuerdo a la recomendación de la OMS, los puntajes del total del test que identifican los distintos niveles de riesgo en la escala del AUDIT son: entre 8 y 15 puntos, consumo de riesgo; entre 16 y 19 puntos, consumo perjudicial y 20 o más puntos o más, dependencia. No obstante lo anterior, la recomendación de la OMS es que la selección de los valores de corte para cada nivel de riesgo en los distintos países debe estar en función de estándares culturales y nacionales (Babor et al., 2001), por lo que debe definirse en estudios específicos en cada país.

En el año 2009 se publicó su validación en Chile en una muestra de 93 individuos (mayores de 15 años) consultantes de un centro de atención primaria, tanto por morbilidad general como por problemas derivados del consumo de alcohol. Además, se incorporó la traducción y adaptación lingüística del instrumento (Alvarado, Garmendia, Acuña, Santis \& Arteaga, 2009). La aplicación del AUDIT se realizó en conjunto con la Entrevista Diagnóstica Internacional Compuesta (CIDI 2.1), como criterio diagnóstico de consumo perjudicial y dependencia de alcohol. Se concluyó que el instrumento es válido y confiable (alfa de Cronbach de 0,83 ) para el tamizaje de las distintas categorías de consumo de alcohol en la población adulta chilena. En base a la sensibilidad y especificidad para cada categoría diagnóstica, se establecieron los siguientes puntos de corte en la escala: entre 6 y 8 puntos, consumo de riesgo y 9 o más puntos, consumo perjudicial o dependencia. Para este estudio se determinó un puntaje menor a 6 puntos como criterio de inclusión.

Conflict Tactics Scales-2 (CTS-2; Straus et al., 1996). Se evaluó previamente mediante este instrumento a ambos grupos en relación al ejercicio de la violencia hacia su pareja, con el fin de asegurar la distinción de dos grupos de hombres: los que ejercen violencia hacia su pareja y los que no. Es un instrumento autoaplicado que mide el grado en que los miembros de una pareja se ven envueltos en ataques físicos o psicológicos mutuamente, así como el uso de razonamiento y negociación para resolver conflictos (Straus et al., 1996). Consta de 78 ítems (39 para cada miembro de la pareja) y contiene cinco subescalas (violencia física, coacción sexual, agresión psicológica, daños y negociación), que se dividen, a su vez, en dos subescalas: "cognitiva" y "emocional" para negociación y "menor" o "severa" para las demás escalas.

Algunos ejemplos de los reactivos son: "Mostré a mi pareja que me preocupaba por ella incluso cuando no estábamos de acuerdo" (escala de Negociación); "Salí dando un portazo de la habitación o de la casa durante una discusión" (escala de agresión psicológica); "Golpeé a mi pareja con algo que le pudo hacer daño" (escala de violencia física); "Utilicé amenazas para obligar a mi pareja a practicar sexo oral o anal conmigo" (escala 
de coacción sexual) y "Perdí el conocimiento por un golpe en la cabeza en una pelea con mi pareja" (escala de daños).

La prueba consta de ocho alternativas de respuesta. Los valores 0 a 6 corresponden a la frecuencia con la que pudo haber ocurrido una conducta: 1 (una vez), 2 (dos veces), 3 (de tres y cinco veces), 4 (de seis a 10 veces), 5 (de 11 a 20 veces) y 6 (más de 20 veces). El 7 se refiere a nunca el año pasado, pero sí antes, y el 0 significa nunca ha ocurrido. Para los fines de este estudio, se consideró violencia cualquier valor diferente de 0 .

El tiempo para completar la escala oscila entre 10 y 15 minutos.

Este trabajo centró la atención únicamente en la violencia masculina dirigida hacia las mujeres, por lo que se analizaron solo los resultados de los 39 reactivos correspondientes.

El instrumento completo posee una consistencia interna, medida por alfa de Cronbach, de 0,94, de acuerdo a los resultados de Barría Muñoz (2014). Por su parte, los 39 ítems correspondientes al agresor masculino presentan una consistencia interna alta $(0,89)$, pese a que la de las cinco subescalas oscila entre 0,59 y 0,84: la mayor se encuentra en la violencia física $(0,84)$, seguida por la agresión psicológica $(0,83)$, coerción sexual $(0,81)$, negociación $(0,77)$ y daños $(0,59)$. Asimismo, Dietz y Jasinski (2007) proponen que la confiabilidad test-retest en agresores de pareja oscila entre 0,80 en la subescala de daños y 0,49 en la de negociación; en las subescalas de violencia, la mayor confiabilidad corresponde a la psicológica $(0,72)$, pero la física y sexual superan también el 0,65. El análisis del efecto del orden de los ítems indica que el formato de presentación es adecuado, en especial si el objetivo es el análisis de la violencia física (Dietz \& Jasinski, 2007).

De acuerdo con el análisis de validez realizado en Chile por Barría Muñoz (2014) en 731 maltratadores y 100 hombres que no habían ejercido violencia hacia su pareja, el instrumento permite discriminar población de hombres no maltratadores y maltratadores, estableciéndose puntuaciones de corte para que puedan ser usadas como valores de referencia nacional. La validación se realizó contrastando información de la CTS-2 con el Cuestionario General sobre Violencia de Barría Muñoz y Macchiavello Rodríguez (2012) y el cuestionario The Spousal Assault Risk Assessment de Kropp y Hart (2000).

Escala de Experiencia en Relaciones Cercanas (ERC). Es un cuestionario auto-aplicable cuyo objetivo es la evaluación del apego adulto en las relaciones amorosas. Originalmente fue creada por Brennan et al. (1998) y, posteriormente, fue revisada (ERC-R) por Fraley, Waller y Brennan (2000). El tiempo de aplicación es de 20 minutos.

La ERC-R es una prueba que, desde un punto de vista cuantitativo y multidimensional, aborda el estudio del sistema comportamental del apego. Este auto-informe evalúa dos dimensiones del apego en una relación de pareja: la dimensión de Ansiedad (ejemplo: "Mi pareja me hace dudar de mí mismo/a") y la dimensión de Evitación (ejemplo: "Prefiero no mostrar a mi pareja mis posibles sentimientos de debilidad o flaqueza").

Cada dimensión corresponde a una escala, las cuales constan de 18 ítems cada una, evaluados en una escala Likert que oscila entre 1 (Totalmente en desacuerdo) a 7 (Totalmente de acuerdo). De la combinación de ambas escalas se definen cuatro estilos de apego. El apego seguro (bajos niveles de ansiedad y evitación) presenta alta autoestima, confianza en los otros y relaciones íntimas cercanas y autónomas. El estilo de apego inseguro temeroso (altos niveles de ansiedad y evitación) muestra una autoimagen negativa, falta de confianza en los demás y un elevado nivel de estrés en las relaciones. El estilo de apego inseguro preocupado (alta ansiedad y baja evitación) evidencia una autoestima dependiente de la opinión y aceptación de los demás. Finalmente, el estilo de apego inseguro desentendido (baja ansiedad y alta evitación) se caracteriza por una visión de sí mismo idealizada, una negación de los sentimientos de estrés y una descalificación de las relaciones íntimas (Bartholomew \& Horowitz, 1991).

Fraley et al. (2000) no sugieren un puntaje general, sino escalas dicotomizadas en alta y baja. La forma de asignar un valor relativo a las dos escalas es asignar percentiles, señalando alta (+) o baja (-) ansiedad o evitación. El punto de corte es 54 puntos para la escala Evitación y 68 puntos para la escala Ansiedad. En el caso de contar con una muestra pequeña, es posible agrupar los resultados de manera dicotómica, apego seguro/inseguro, para marcar más claramente las eventuales diferencias. Este último fue el sistema utilizado en este estudio.

Los índices de consistencia interna, reportados por Brennan et al. (1998) mediante alfa de Cronbach, son 0,91 y 0,94 para las escalas Ansiedad y Evitación, respectivamente. La evidencia de validez de constructo de la ERC está basada en estudios que muestran que es un predictor significativo de constructos teóricamente relevantes, tales como la satisfacción marital (Brennan et al., 1998) y la presencia de problemas 
interpersonales (Wei, Vogel, Ku \& Zakalik, 2005). De acuerdo con el análisis de validez del instrumento en Chile, Spencer, Guzmán, Fresno y Ramos (2013) afirman que el análisis de validez de constructo indica que en términos generales los puntajes de las escalas de la versión chilena de la ERC se relacionan de la manera esperada con el cuestionario de apego adulto CaMir (Garrido, Santelices, Pierrehumbert \& Armijo, 2009).

En este estudio se utilizó la versión chilena validada por Spencer et al. (2013).

Test Reading the Mind of the Eyes (Baron-Cohen et al., 1997, 2001). El test mide mentalización en adultos y consta de 36 imágenes en blanco y negro de la parte superior del rostro (ojos y cejas) de personas de ambos sexos. El sujeto debe "leer la mirada" y elegir entre cuatro respuestas posibles, marcando la que para él represente el estado mental de la imagen. Este instrumento analiza el procesamiento mental en tres niveles: (a) percepción no consciente, automática, de una mirada; (b) el otorgamiento de una valencia a esa percepción y (c) y el procesamiento consciente para elegir entre las cuatros opciones. Se otorga un punto por cada respuesta correcta, sumando un máximo de 36 puntos. Al no haber normas estandarizadas para Chile, en este estudio se utilizaron las normas propuestas por Román et al. (2012) recogidas de la población de Buenos Aires, Argentina. Estas definen que la media esperada en hombres adultos es de $23(D E=4,8)$. De este modo, el rango normal queda establecido de 23-30 aciertos y una puntuación superior a 30 indica un elevado nivel de mentalización.

El índice de consistencia interna es de 0,91, de acuerdo a alfa de Cronbach, en estudios correlacionales (Baron-Cohen et al., 1997) realizados con grupos mixtos (hombres y mujeres). En relación a la validez, los autores han realizado múltiples estudios (BaronCohen et al., 1997, 2001), comparando los resultados de este instrumento con otros instrumentos válidos, como el Test de la Empatía Cociente (EQ) de Baron-Cohen y Wheelwright (2004) y la Escala de Alexitimia de Toronto (TAS) de Taylor, Ryan y Bagby (1985), encontrando correlaciones entre ellos $(p<0,01)$.

\section{Procedimiento}

Se realizó una aplicación piloto de los instrumentos a cinco sujetos usuarios del hospital seleccionado a fin de ajustar el protocolo y evaluar los tiempos de aplicación.

Los participantes del grupo de estudio fueron invitados a participar de la investigación durante la primera entrevista psicológica para el control de impulsos en la unidad de salud mental del hospital seleccionado. Los del grupo de comparación fueron reclutados a través de la derivación de los médicos tratantes y/o enfermeras de los asistentes al consultorio de especialidades del mismo hospital.

Todos los sujetos participaron de forma voluntaria y firmaron una hoja de consentimiento informado. La investigación contó con la aprobación del comité de ética del hospital.

La evaluación fue realizada individualmente por la primera autora, quien realizó la totalidad de las entrevistas a ambos grupos de manera paralela en el periodo comprendido entre Marzo y Septiembre de 2014. Se aplicó en primer lugar la entrevista sociodemográfica y luego, la batería de instrumentos del estudio, partiendo por los instrumentos de screening, el AUDIT y la CTS-2, a fin de asegurar que no existiera consumo de alcohol y separar efectivamente un grupo de estudio y uno de comparación, respectivamente. Luego se aplicó la ERC-R y el test Reading the Mind of the Eyes para evaluar las variables del estudio.

La batería de instrumentos fue digitalizada y organizada en un programa de computación (Google Drive), pidiéndoles a los participantes que respondieran directamente en el computador.

\section{Análisis de Datos}

Se empleó estadística descriptiva, específicamente análisis no paramétricos, debido a que la muestra era pequeña y no probabilística. Se aplicó la prueba de $\chi^{2}$ para evaluar diferencias en la frecuencia del tipo de apego (seguro/inseguro) y en la del tipo de apego inseguro (preocupado/rechazante/temeroso) en cada grupo, utilizándose en el segundo análisis la prueba exacta de Fisher, por existir frecuencias esperadas menores a cinco. También se aplicó la prueba $U$ de Mann-Whitney para muestras independientes para comparar los promedios de los grupos en las dimensiones de violencia (física/sexual/psicológica/daños/negociación), estilos de apego (ansiedad/evitación) y mentalización.

Para los análisis de datos se utilizó el programa SPSS versión 22.0. 


\section{Resultados}

\section{Tipo de Violencia}

Los resultados de la CTS- 2 permiten concluir que las personas del grupo de estudio puntúan más alto que el grupo de comparación en cuatro de cinco subescalas: Violencia Física, $U=99, Z=-2,780, p=0,006$, Coacción Sexual, $U=128, Z=-2,301, p=0,052$, Agresión Psicológica, $U=126, Z=-2,096, p=0,046, \mathrm{y}$ Negociación, $U=100,5, Z=-2,469, p=0,014$. La escala de Daños no presenta variación entre ambos grupos, $U=168,5, Z=-1,483, p=0,398$. Los promedios y desviaciones estándar pueden verse en la Tabla 1 . Estos resultados confirman que la CTS-2 es un instrumento válido para la evaluación del ejercicio de la violencia, como proponen diferentes investigaciones en el área (Loinaz et al., 2012; Mora Ríos, Natera Rey, Tiburcio Sáinz \& Juárez, 2008). Asimismo, asegura la distinción de los dos grupos de hombres, estudio y comparación.

Tabla 1

Tipo de Violencia, Según Grupo (Promedio y Desviación Estándar)

\begin{tabular}{lcccc}
\hline \multirow{2}{*}{ Tipo de violencia } & \multicolumn{2}{c}{ Experimental } & \multicolumn{2}{c}{ Comparación } \\
\cline { 2 - 5 } & $M$ & $D E$ & $M$ & $D E$ \\
\hline Violencia física & 7,02 & 3,00 & 4,02 & 1,99 \\
Coacción sexual & 2,05 & 1,38 & 0,95 & 0,37 \\
Agresión psicológica & 4,50 & 2,79 & 1,05 & 1,78 \\
Daños & 1,75 & 1,28 & 1,25 & 0,27 \\
Negociación & 11,23 & 4,33 & 8,23 & 3,32 \\
\hline
\end{tabular}

\section{Apego}

Existen diferencias entre ambos grupos en el tipo de apego, $\chi^{2}(1, N=40)=3,636, p=0,004$, con un porcentaje mayor de apego inseguro en el grupo experimental (60\%) que en el grupo de comparación (30\%). Hay un $40 \%$ de apego seguro en el grupo experimental, mientras que el $60 \%$ restante corresponde al grupo de comparación.

En relación a las dimensiones del apego, existe diferencia entre los promedios de los grupos en la variable ansiedad, $U=93,5, Z=-2,882, p=0,004$; no así en la de evitación, $U=164,5, Z=-0,961, p=0,336$ (ver Tabla 2).

Tabla 2

Tipo de Apego, Según Grupo (Promedio y Desviación Estándar)

\begin{tabular}{lcccc}
\hline \multirow{2}{*}{ Tipo de apego } & \multicolumn{2}{c}{ Experimental } & \multicolumn{2}{c}{ Comparación } \\
\cline { 2 - 5 } & $M$ & $D E$ & $M$ & $D E$ \\
\hline Ansiedad & 55,40 & 20,86 & 53,44 & 19,85 \\
Evitación & 51,30 & 14,29 & 48,30 & 13,28 \\
\hline
\end{tabular}

Asimismo, es posible observar diferencias entre los grupos en los distintos tipos de apego inseguro, $\chi^{2}(2$, $N=18)=6,136, p=0,031$, como es posible observar en la Tabla 3. Estas diferencias se confirman a través del estadístico exacto de Fisher, $p=0,049$. De los que tienen apego inseguro, el grupo experimental presenta mayor apego temeroso y el grupo de comparación, mayor apego preocupado. 
Tabla 3

Estilo de Apego, Según Grupo

\begin{tabular}{lrrrrrr}
\hline & \multicolumn{2}{c}{$\begin{array}{c}\text { Experimental } \\
\text { Estilo de apego }\end{array}$} & \multicolumn{1}{c}{$\begin{array}{c}\text { Comparación } \\
(n=20)\end{array}$} & \multicolumn{2}{c}{ Total } \\
\cline { 2 - 7 } & \multicolumn{1}{c}{$f$} & \multicolumn{1}{c}{$\%$} & \multicolumn{1}{c}{$f$} & \multicolumn{1}{c}{$\%$} & \multicolumn{1}{c}{$f$} & $\%$ \\
\hline Apego Seguro (BA + BE) & 8 & 40,0 & 14 & 70,0 & 22 & 55,0 \\
Apego Inseguro & 12 & 60,0 & 6 & 30,0 & 18 & 45,0 \\
- Apego Rechazante (BA + AE) & 5 & 41,7 & 3 & 50,0 & 8 & 44,4 \\
- Apego Preocupado (AA + BE) & 3 & 25,0 & 3 & 50,0 & 6 & 33,3 \\
- Apego Temeroso (AA + AE) & 4 & 33,3 & 0 & 0,0 & 4 & 22,2 \\
\hline
\end{tabular}

Nota. BA: baja ansiedad, AA: alta ansiedad, BE: baja evitación, AE: alta evitación.

\section{Mentalización}

Los resultados de la evaluación de mentalización indican puntajes similares entre ambos grupos, $U=$ $177,5, Z=-0,611, p=0,547$, como se muestra en la Tabla 4 .

Tabla 4

Mentalización Según Grupo

\begin{tabular}{lccc}
\hline \multicolumn{1}{c}{ Grupo } & $M$ & $\begin{array}{c}\text { Puntaje normal } \\
\text { esperado }\end{array}$ & $D E$ \\
\hline Estudio & 19,50 & 23 & 0,96 \\
Comparación & 20,30 & 23 & 1,11 \\
\hline$N=40$ & &
\end{tabular}

\section{Discusión}

El grupo de comparación mostró un mayor porcentaje de apego seguro que el grupo de estudio, lo que apoya la primera hipótesis que plantea que existen diferencias en el estilo de apego entre los hombres que presentan antecedentes de violencia en la pareja de los que no la han ejercido. Estas diferencias apuntarían a que los hombres que han ejercido violencia presentan más un estilo de apego inseguro que los hombres que no tienen antecedentes de aquello.

Si bien los autores proponen que los hombres que han ejercido violencia pueden presentar apego seguro (Holtzworth-Munroe et al., 2003; Holtzworth-Munroe \& Stuart, 1994; Lawson et al., 2006; Waltz et al., 2000), en la investigación de Buck et al. (2014) los porcentajes fluctúan entre un 15 y un 27\% como máximo. Los datos en Chile reportan un 21,7\% (Barría Muñoz, 2013). Es por ello que llama la atención que el 40\% de hombres que han ejercido violencia en este estudio presente apego seguro. Es probable que las condiciones contextuales en las cuales fueron reclutados estos hombres tenga alguna incidencia en el aumento de este porcentaje, es decir, son hombres que han sido condenados por lesiones menos graves y que no se encuentran cumpliendo la pena en cárceles, como la mayoría de los participantes de los estudios de este tipo y desde donde surgen, en su mayoría, los porcentajes de referencia (Carrasco-Portiño, Vives-Cases, Gil-González \& Álvarez-Dardet, 2007; Echeburúa et al., 2009; Echeburúa, Fernández-Montalvo \& Amor, 2006; HoltzworthMunroe et al., 2000; Holtzworth-Munroe \& Stuart, 1994; Jacobson \& Gottman, 1998/2001; Loinaz Calvo, Irureta Lecumberri \& Doménech Burset, 2011; Loinaz et al., 2010). Por ello, parece relevante generar investigación en hombres que ejercen violencia en contextos domésticos no carcelarios, ya que aparentemente presentan características diferentes.

En las características del apego en hombres que han ejercido violencia, los resultados permiten señalar que la ansiedad en las relaciones interpersonales es un factor de riesgo para el ejercicio de la violencia por 
parte de los hombres. Estos resultados pueden ser explicados a la luz de lo señalado por los teóricos que han profundizado en este tipo de apego. El apego en el adulto se manifiesta en la calidad de las relaciones y en la regulación del afecto ante situaciones de amenaza o conflicto (Feeney, Noller \& Roberts, 2000; Hazan \& Shaver, 1987). En lo que respecta a las situaciones activadoras del sistema de apego en la edad adulta, Kobak y Duemmler (1994) describieron tres situaciones: el miedo, que motiva la búsqueda de refugio emocional; los retos, que motivan la búsqueda de una base segura, y los conflictos en las relaciones, que motivan la búsqueda de proximidad. Estresores leves no evocan los comportamientos de apego en la edad adulta, ya que el sujeto puede confiar en la disponibilidad de la figura de apego, a pesar de una ausencia de proximidad física (Hinde \& Stevenson-Hinde, 1976). Sin embargo, frente a situaciones conflictivas difíciles, los hombres que presentan ansiedad frente al abandono tienen mayor probabilidad de ejercer violencia hacia su pareja, en comparación con los hombres que no presentan esta cualidad. Feeney (1999) apoya esta tesis, basándose en los resultados de su trabajo clínico. En este proceso descubre que los individuos con mayor ansiedad sobre la relación reportan mayor grado de conflicto, sugiriendo que los motores serían fundamentalmente la inseguridad básica y el temor al abandono. Del mismo modo, este grupo tiende a la resolución de conflictos de manera más coercitiva y suspicaz, generando justamente los resultados y situaciones que más temen (Feeney, 1999). Por su parte, Babcock et al. (2000) hipotetizan que la conducta de hombres con preponderancia de ansiedad en el apego puede explicarse como una descarga emocional que mantiene, momentáneamente, la proximidad de la pareja, como respuesta al temor de ser abandonados. Es posible concluir, entonces, que hombres que presentan mayor ansiedad en las relaciones, caracterizada por la necesidad de una mayor cercanía afectiva, preocupación por la pérdida del amor del otro, temor al rechazo y al abandono y mayor inseguridad frente a los afectos podrían ser más proclives a que, frente a una situación de conflicto en la cual sientan rechazo por parte del otro y un temor real al término de la relación, se active el sistema de apego de la infancia y agredan a su pareja como un modo de representar estos temores y, a su vez, impedir que el otro lo abandone, como hacen los niños frente a la amenaza de un abandono real o imaginado.

El hecho de que entre los participantes que no habían ejercido violencia no existiera el apego temeroso — coincidente con el apego desorganizado de Main y Solomon (1990)_ fortalece las teorías que explican que el apego desorganizado se relaciona cercanamente con el ejercicio de la violencia por parte de los hombres (Lyons-Ruth et al., 1993, 1997; Main \& Solomon, 1990; Moss et al., 1996). Este hallazgo podría explicar algunas conductas presentes en los hombres que han ejercido violencia hacia sus parejas, que se relaciona, entre otros aspectos, con la necesidad imperiosa de controlar al otro (Lyon-Ruth et al., 1993). En términos relacionales, en la persona con apego temeroso las experiencias de rechazo no ocasionarían un descontrol extremo; sin embargo, la hiperactivación del sistema de apego derivaría en una incapacidad para analizar racionalmente las experiencias que el adulto desorganizado considera como amenazantes. Cuanta más implicación e intimidad existan en una relación mayor es la probabilidad de que se pongan al descubierto muchas de las dinámicas de relación que se aprendieron en las interacciones con los progenitores. Si estas nuevas experiencias confirman las expectativas, es probable que el modelo infantil, en lugar de modificarse, se refuerce (Melero \& Cantero, 2008). En este sentido, los hombres que presentan este tipo de apego desorganizado y que presentan una amplia implicación de intimidad con su pareja, frente a situaciones afectivas amenazantes, pueden agredir a su pareja, impedidos de analizar racionalmente la situación que los provoca.

Respecto de la mentalización, los hallazgos obtenidos dan cuenta de que no existirían diferencias en la mentalización en hombres condenados por violencia de aquellos que no presentan antecedentes de haber ejercido violencia hacia su pareja. Es decir, hombres que han ejercido violencia y los que no la han ejercido poseen niveles de mentalización similares. Estos hallazgos son divergentes a los aportes de los autores que proponen que los hombres que ejercen violencia tendrían una insuficiente capacidad de mentalización (Fonagy, 2003; Fonagy \& Target, 2008; Jenkins, 1990; Lyons-Ruth, 1996; Walker, 1979). A la luz de la segunda hipótesis que se propuso en este estudio, los niveles similares de mentalización en ambos grupos de hombres, al ser contradictorio con la tesis que postula que en hombres que han ejercido violencia existiría un déficit en la mentalización, genera algunas preguntas que se desprenden de este trabajo: ¿Será que en hombres que ejercen violencia hacia sus parejas existe una función específica de la mentalización que presente déficit? En el caso de estos hombres ¿podrá actuar la mentalización como una variable mediadora o modeladora de la violencia? Y si es así, ¿será posible que exista un mecanismo que, bajo situaciones de alto estrés en la pareja, este actúe?

Es importante destacar que no solo los puntajes en mentalización han sido similares en ambos grupos, sino también que estos puntajes se encuentran en ambos grupos por debajo del puntaje normativo establecido 
para la mentalización en Latinoamérica por Román et al. (2012), que es de 23 puntos con una desviación estándar de 4,8. Es posible comprender este fenómeno desde la perspectiva del efecto del contexto sociocultural en los niveles de mentalización en los hombres. La mentalización o función reflexiva se refiere a una variada serie de operaciones psicológicas que incluye un componente auto-reflexivo y un componente interpersonal. El énfasis en su desarrollo teórico ha estado en la perspectiva intrapsíquica, individual y relacional (Lanza Castelli \& Bilbao Bilbao, 2012). Sin embargo, para su desarrollo es fundamental el lenguaje y, con ello, la historia social y material de la cultura (Lanza Castelli \& Bilbao Bilbao, 2012), las cuales son conceptos y sistemas simbólicos desarrollados para interactuar con el entorno. Desde esta perspectiva, los diferentes abordajes sobre mentalización aún parecen ser insuficientes, en el sentido de que, a pesar de existir propuestas que toman en cuenta el contexto sociocultural (Roman et al., 2012), estas siguen tratando la mentalización como un fenómeno sobre todo individual y relacional. Una posible proyección de este estudio se relaciona con la posibilidad de evaluar diferencias socioculturales y socioeconómicas en el contexto chileno en términos de mentalización, aspecto que no ha sido abordado empíricamente hasta ahora.

A modo de conclusión, comprendiendo que las dimensiones del apego (ansiedad y evitación) son determinantes en el modo en que las personas se relacionan entre sí en condiciones de intimidad afectiva, se podría concluir que, al presentar bajos indicadores de evitación en sus relaciones, los hombres que son condenados por violencia de tipo leve hacia sus parejas logran establecer relaciones cercanas con otro y son capaces de ser receptivos e involucrarse íntimamente en los vínculos; sin embargo, en situaciones de alta interdependencia en sus relaciones, experimentarían un alto nivel de ansiedad respecto de la posibilidad de ser rechazado o abandonado, al poseer una visión negativa respecto de sí mismo y de la propia valía.

Finalmente, este estudio posee algunas limitaciones que deben ser consideradas. Una de ellas se relaciona con las características del grupo de comparación. Si bien en este estudio los participantes de este grupo puntuaron bajo en la CTS-2, indicando la baja presencia de violencia hacia su pareja, no es posible contar con el referente de la pareja actual o pasada y comprobar estos resultados con su contraparte. Asimismo, la deseabilidad social puede ser un factor que esté afectando al estilo de respuesta en ambas muestras (agresores y controles). Por otra parte, en términos metodológicos, es importante señalar una limitación del test de Baron-Cohen et al. (1997), la que ellos mismos plantean como una dificultad: dado que el Test de la Mirada implica asignar una palabra a una imagen, no está claro si los problemas de puntaje en ocasiones se pueden relacionar con la comprensión de las palabras mismas que se presentan para la elección de la imagen, pese a contar con un glosario de términos de cada elemento. Por otro lado, hay que agregar que si bien el test posee validación en relación a otros instrumentos que miden mentalización, aún no se ha adaptado a la población chilena. Esta situación pudiese también tener una incidencia en los resultados en la mentalización. Por último, tanto el tamaño como el tipo de muestra no permiten generalizar estos resultados. Ampliar el tamaño de la muestra en futuras investigaciones permitiría corroborar relaciones en las variables del apego adulto y el ejercicio de la violencia que en esta investigación han sido desestimadas por su baja significación, como, por ejemplo, la relación en el grupo de estudio entre la escala de coerción sexual y apego inseguro.

\section{Referencias}

Allen, J. G. (2006). Mentalizing in practice. En J. G. Allen \& P. Fonagy (Eds.), Handbook of mentalization-based treatment (pp. 3-30). Chichester, Reino Unido: John Wiley \& Sons.

Alvarado, M. E., Garmendia, M. L., Acuña, G., Santis, R. \& Arteaga, O. (2009). Validez y confiabilidad de la versión chilena del Alcohol Use Disorders Identification Test (AUDIT). Revista Médica de Chile, 137, 1463-1468. https://doi.org/10.4067/S003498872009001100008

American Psychological Association (1996). Violence and the family: Report of the American Psychological Association Presidential Task Force on violence and the family. Washington, DC: Autor.

Amor, P. J., Echeburúa, E. \& Loinaz, I. (2009). ¿Se puede establecer una clasificación tipológica de los hombres violentos contra su pareja? International Journal of Clinical and Health Psychology, 9, 519-539.

Ansbro, M. (2008). Using attachment theory with offenders. Probation Journal, 55, 231-244. https://doi.org/10.1177/0264550508092812

Babcock, J. C., Jacobson, N. S., Gottman, J. M. \& Yerington, T. P. (2000). Attachment, emotional regulation, and the function of marital violence: Differences between secure, preoccupied, and dismissing violent and nonviolent husbands. Journal of Family Violence, 15, 391-409. https://doi.org/10.1023/A:1007558330501

Babor, T. F., Higgins-Biddle, J. C., Saunders, J. B. \& Monteiro, M. G. (2001). AUDIT, cuestionario de identificación de los trastornos debidos al consumo de alcohol: pautas para su utilización en atención primaria. Genève: Suiza: Organización Mundial de la Salud.

Baron-Cohen, S., Jolliffe, T., Mortimore, C. \& Robertson, M. (1997). Another advanced test of theory of mind: Evidence from very high functioning adults with autism or Asperger syndrome. Journal of Child Psychology and Psychiatry, 38, 813-822. https://doi.org/10.1111/j.1469-7610.1997.tb01599.x 
Baron-Cohen, S., Wheelwright, S., Hill, J., Raste, Y. \& Plumb, I. (2001). The "Reading the Mind in the Eyes" test revised version: A study with normal adults, and adults with Asperger syndrome or high-functioning autism. Journal of Child Psychology and Psychiatry, 42, 241-251. https://doi.org/10.1111/1469-7610.00715

Baron-Cohen, S. \& Wheelwright, S. (2004). The Empathy Quotient: An investigation of adults with Asperger syndrome or high functioning autism, and normal sex differences. Journal of Autism and Developmental Disorders, 34, $163-175$. https://doi.org/10.1023/B:JADD.0000022607.19833.00

Barría Muñoz, J. R. (2013). Aproximación a un modelo teórico de tipologías de hombres maltratadores. El hombre agresor de su pareja: un actor desconocido (Tesis de Doctorado, Universidad de Chile, Santiago, Chile). Extraído de http://repositorio.uchile.cl/ bitstream/handle/2250/114808/barrias_j.pdf?sequence=1\&isAllowed=y

Barría Muñoz, J. (2014). Escala de Tácticas de Conflicto (CTS-2) (Conflict Tactics Scales) en una muestra chilena de hombres maltratadores: su validez y confiabilidad. Santiago, Chile: Universidad Andrés Bello. Extraído de https://www.researchgate.net/publication/268150400_Escala_de_Tacticas_de_Conflicto_CTS-2 _Conflict_Tactics_Scales_en_una_muestra_chilena_de_Hombres_Maltratadores_su_validez_y_confiabilidad_Conflict_Tactics_ Scale_CTS-2_in_a_sample_of_Chilean_Men_Battere

Barría Muñoz, J. R. \& Macchiavello Rodríguez, A. (2012). Anatomía de los hombres que ejercen violencia hacia sus parejas: primer levantamiento de datos para el diseño de un perfil. Psicologia.com, 16, artículo 8. Extraído de http://www.psiquiatria.com/ bibliopsiquis/handle/10401/5511

Bartholomew, K. \& Horowitz, L. M. (1991). Attachment styles among young adults: A test of a four-category model. Journal of Personality and Social Psychology, 61, 226-244. doi:10.1037/0022-3514.61.2.226

Barudy, J. (1998). El dolor invisible en la infancia: una lectura ecosistémica del maltrato infantil. Barcelona, España: Paidós Ibérica.

Blázquez Alonso, M., Moreno Manso, J. M. \& García-Baamonde Sánchez, M. E. (2010). Revisión teórica del maltrato psicológico en la violencia conyugal. Psicología y Salud, 20, 65-75.

Bonino, L. (2008). Hombres y violencia de género. Más allá de los maltratadores y de los factores de riesgo. Madrid, España: Ministerio de Trabajo e Inmigración, Subdirección General de Información Administrativa y Publicaciones.

Bowlby, J. (1969/1976). El vínculo afectivo (I. Pardal, Trad.; Título original: Attachment and loss, Volume I: Attachment). Buenos Aires, Argentina: Paidós.

Bowlby, J. (1980/1984). La pérdida afectiva: tristeza y depresión (A. Báez, Trad.; Título original: Attachment and loss, Volume III: Loss, sadness, and depression). Buenos Aires, Argentina: Paidós.

Bowlby, J. (1988). A secure base: Clinical applications of attachment theory. Abingdon, Reino Unido: Routledge.

Brennan, K. A., Clark, C. L. \& Shaver, P. R. (1998). Self-report measurement of adult attachment: An integrative overview. En J. A. Simpson \& W. S. Rholes (Eds.), Attachment theory and close relationships (pp. 46-76). New York, NY: Guilford Press.

Buck, N. M. L., Leenaars, P. E. M., Emmelkamp, P. M. G. \& van Marle, H. J. C. (2014). Personality traits are related to intimate partner violence among securely attached individuals. Journal of Family Violence, 29, 235-246. https://doi.org/10.1007/s10896-014-95847

Capaldi, D. M. \& Kim, H. K. (2007). Typological approaches to violence in couples: A critique and alternative conceptual approach. Clinical Psychology Review, 27, 253-265. https://doi.org/10.1016/j.cpr.2006.09.001

Carrasco-Portiño, M., Vives-Cases, C., Gil-González, D. \& Álvarez-Dardet, C. (2007). ¿Qué sabemos sobre los hombres que maltratan a su pareja? Una revisión sistemática. Revista Panamericana de Salud Pública, 22, 55-63. https://doi.org/10.1590/S102049892007000600008

Chile, Ministerio de Justicia (2016). Establece ley de violencia intrafamiliar. Santiago, Chile: Biblioteca del Congreso Nacional de Chile. Extraído de http://www.leychile.cl/Navegar?idNorma=242648

Chile, Ministerio de Trabajo y Previsión Social (2017). Establece subsidio familiar para personas de escasos recursos y modifica normas que indica. Santiago, Chile: Biblioteca del Congreso Nacional de Chile. Extraído de http://www.leychile.cl/Navegar?idNorma=29448

Dietz, T. L. \& Jasinski, J. L. (2007). The effect of item order on partner violence reporting: An examination of four versions of the revised Conflict Tactics Scales. Social Science Research, 36, 353-373. https://doi.org/10.1016/j.ssresearch.2006.04.006

Dolan, M. \& Fullam, R. (2004). Theory of mind and mentalizing ability in antisocial personality disorders with and without psychopathy. Psychological Medicine, 34, 1093-1102. https://doi.org/10.1017/S0033291704002028

Doumas, D. M., Pearson, C. L., Elgin, J. E. \& McKinley, L. L. (2008). Adult attachment as a risk factor for intimate partner violence: The "mispairing" of partners' attachment styles. Journal of Interpersonal Violence, 23, 616-634. https://doi.org/10.1177/0886260507313526

Dutton, D. G. (2008). Blended behavior therapy for intimate violence. En A. C. Baldry \& F. W. Winkel (Eds.), Intimate partner violence prevention and intervention: The risk assessment and management approach (pp. 133-146). New York, NY: Nova Science.

Dutton, D. G. (2011). Attachment and violence: An anger born of fear. En P. R. Shaver \& M. Mikulincer (Eds.), Human aggression and violence: Causes, manifestations, and consequences (pp. 259-275). Washington, DC: American Psychological Association.

Dutton, D. G. \& Golant, S. K. (1995/1997). El golpeador: un perfil psicológico (A. Negrotto, Trad.; Título original: The batterer: A psychological profile). Barcelona, España: Paidós.

Echeburúa, E., Amor, P. J. \& de Corral, P. (2009). Hombres violentos contra la pareja: trastornos mentales y perfiles tipológicos. Pensamiento Psicológico, 6, 27-36.

Echeburúa, E., Fernández-Montalvo, J. \& Amor, P. J. (2006). Psychological treatment of men convicted of gender violence: A pilot- study in Spanish prisons. International Journal of Offender Therapy and Comparative Criminology, 50, 57-70. https://doi.org/ $10.1177 / 0306624 X 05277662$

Feeney, J. A. (1999). Adult romantic attachment and couple relationships. En J. Cassidy \& P. R. Shaver (Eds.), Handbook of attachment: Theory, research, and clinical applications (pp. 355-377). New York, NY: Guilford Press.

Feeney, J. \& Noller, P. (1996/2001). Apego adulto (Título original: Adult attachment). Bilbao, España: Desclée de Brouwer.

Feeney, J. A., Noller, P. \& Roberts, N. (2000). Attachment and close relationships. En C. Hendrick \& S. S. Hendrick (Eds.), Close relationships: A sourcebook (pp. 185-201). Thousand Oaks, CA: SAGE.

Fonagy, P. (2003). Towards a developmental understanding of violence. The British Journal of Psychiatry, 183, 190-192. https://doi.org/ 10.1192/bjp.183.3.190

Fonagy, P. \& Bateman, A. W. (2006). Mechanisms of change in mentalization-based treatment of BPD. Journal of Clinical Psychology, 62, 411-430. https://doi.org/10.1002/jclp.20241 
Fonagy, P. \& Target, M. (1997). Attachment and reflective function: Their role in self-organization. Development and Psychopathology, 9, 679-700. https://doi.org/10.1017/S0954579497001399

Fonagy, P. \& Target, M. (2008). Attachment, trauma and psychoanalysis: Where psychoanalysis meets neuroscience. En E. L. Jurist, A. Slade \& S. Bergner (Eds.), Mind to mind: Infant research, neuroscience and psychoanalysis (pp. 15-49). New York, NY: Other Press.

Fonagy, P., Target, M. \& Gergely, G. (2000). Attachment and borderline personality disorder: A theory and some evidence. Psychiatric Clinics of North America, 23, 103-122. https://doi.org/10.1016/S0193-953x(05)70146-5

Fonagy, P., Target, M., Steele, H. \& Steele, M. (1998). Reflective-functioning manual, version 5.0, for application to adult attachment interviews. London, Reino Unido: University College London.

Fraley, R. C. \& Shaver, P. R. (2000). Adult romantic attachment: Theoretical developments, emerging controversies, and unanswered questions. Review of General Psychology, 4, 132-154. https://doi.org/10.1037/1089-2680.4.2.132

Fraley, R. C., Waller, N. G. \& Brennan, K. A. (2000). An item response theory analysis of self-report measures of adult attachment. Journal of Personality and Social Psychology, 78, 350-365. https://doi.org/10.1037//0022-3514.78.2.350

Garrido, L., Santelices, M. P., Pierrehumbert, B. \& Armijo, I. (2009). Validación chilena del cuestionario de evaluación de apego en el adulto CAMIR. Revista Latinoamericana de Psicología, 41, 79-96.

Gómez, M., Molina, D. \& Arana, M. (2013). Teoría de la mente en un grupo de personas vinculadas al conflicto armado y en proceso de resocialización. Revista Colombiana de Ciencias Sociales, 4, 244-257.

Guzmán, M. \& Contreras, P. (2012). Estilos de apego en relaciones de pareja y su asociación con la satisfacción marital. Psykhe, 21(1), 69-82. https://.doi.org/10.7764/psykhe.21.1.499

Hamel, J. (2009). Toward a gender-inclusive conception of intimate partner violence research and theory: Part 2 - New directions. International Journal of Men's Health, 8, 41-59. https://doi.org/10.3149/jmh.0601.36

Hazan, C. \& Shaver, P. (1987). Romantic love conceptualized as an attachment process. Journal of Personality and Social Psychology, 52, 511-524. https://doi.org/10.1037/0022-3514.52.3.511

Hinde, R. A. \& Stevenson-Hinde, J. (1976). Towards understanding relationships: Dynamic stability. En P. P. G. Bateson \& R. A. Hinde (Eds.), Growing points in ethology (pp. 451-479). Cambridge, Reino Unido: Cambridge University Press.

Holtzworth-Munroe, A., Meehan, J. C., Herron, K., Rehman, U. \& Stuart, G. L. (2000). Testing the Holtzworth-Munroe and Stuart (1994) batterer typology. Journal of Consulting and Clinical Psychology, 68, 1000-1019. https://doi.org/10.1037/0022006X.68.6.1000

Holtzworth-Munroe, A., Meehan, J. C., Herron, K., Rehman, U. \& Stuart, G. L. (2003). Do subtypes of maritally violent men continue to differ over time? Journal of Consulting and Clinical Psychology, 71, 728-740. https://doi.org/10.1037/0022-006X.71.4.728

Holtzworth-Munroe, A. \& Stuart, G. L. (1994). Typologies of male batterers: Three subtypes and the differences among them. Psychological Bulletin, 116, 476-497. https://doi.org/10.1.1.227.2979

Ibaceta Watson, F. (2004). Hombres que ejercen violencia en la pareja: reflexiones de una experiencia de atención individual. Terapia Psicológica, 22(2), 157-164.

Jacobson, N. \& Gottman, J. M. (1998/2001). Hombres que agreden a sus mujeres: cómo poner fin a las relaciones abusivas (C. Castells \& A. Quiroga, Trads.; Título original: When men batter women: New insights into ending abusive relationships). Barcelona, España: Paidós Ibérica.

Jenkins, A. (1990). Invitations to responsibility: The therapeutic engagement of men who are violent and abusive. Adelaide, Australia: Dulwich Centre. https://doi.org/10.1002/j.1467-8438.1990.tb00796.x

Johnson, M. P. \& Ferraro, K. J. (2000). Research on domestic violence in the 1990s: Making distinctions. Journal of Marriage and Family, 62, 948-963. https://doi.org/10.1111/j.1741-3737.2000.00948.x

Kobak, R. R. \& Duemmler, S. (1994). Attachment and conversation: Toward a discourse analysis of adolescent and adult security. En K. Bartholomew \& D. Perlman (Eds.), Attachment processes in adulthood: Advances in personal relationships (pp. 121-149). London, Reino Unido: Jessica Kingsley.

Kropp, P. R. \& Hart, S. D. (2000). The Spousal Assault Risk Assessment (SARA) guide: Reliability and validity in adult male offenders. Law and Human Behavior, 24, 101-118. https://doi.org/10.1023/A:1005430904495

Lanza Castelli, G. \& Bilbao Bilbao, I. (2012). Un método para la evaluación de la mentalización en el contexto interpersonal. EFPP Psychoanalytic Psychotherapy Review, 02, 1-17.

Lawson, D. M., Barnes, A. D., Madkins, J. P. \& Francois-Lamonte, B. M. (2006). Changes in male partner abuser attachment styles in group treatment. Psychotherapy, 43, 232-237. https://doi.org/10.1037/0033-3204.43.2.232

Lawson, D. M. \& Brossart, D. F. (2009). Attachment, interpersonal problems, and treatment outcome in group therapy for intimate partner violence. Psychology of Men \& Masculinity, 10, 288-301. https://doi.org/10.1037/a0017043

Lawson, D. M. \& Malnar, S. G. (2011). Interpersonal problems as a mediator between attachment and intimate partner violence. Journal of Family Violence, 26, 421-430. https://doi.org/10.1007/s10896-011-9376-2

Loinaz, I., Echeburúa, E. \& Torrubia, R. (2010). Tipología de agresores contra la pareja en prisión. Psicothema, 22, $106-111$.

Loinaz, I., Echeburúa, E. \& Ullate, M. (2012). Estilo de apego, empatía y autoestima en agresores de pareja. Terapia Psicológica, 30(2), 61-70.

Loinaz Calvo, I., Irureta Lecumberri, M. \& Doménech Burset, F. (2011). Análisis de la reincidencia en agresores de pareja (Documentos de Trabajo). Barcelona, España: Generalitat de Catalunya, Departament de Justícia, Centre d'Estudis Jurídics i Formació Especialitzada. Extraído de http://www.recercat.cat/bitstream/handle/2072/171050/SC_3_172_11_cast.pdf?sequence=1

Lyons-Ruth, K. (1996). Attachment relationships among children with aggressive behavior problems: The role of disorganized early attachment patterns. Journal of Consulting and Clinical Psychology, 64, 64-73. https://doi.org/10.1037/0022-006X.64.1.64

Lyons-Ruth, K., Alpern, L. \& Repacholi, B. (1993). Disorganized infant attachment classification and maternal psychosocial problems as predictors of hostile-aggressive behavior in the preschool classroom. Child Development, 64, 572-585. https://doi.org/ 10.1111/j.1467-8624.1993.tb02929.x

Lyons-Ruth, K., Easterbrooks, M. A. \& Cibelli, C. D. (1997). Infant attachment strategies, infant mental lag, and maternal depressive symptoms: Predictors of internalizing and externalizing problems at age 7. Developmental Psychology, 33, 681-692. https://doi.org/10.1037/0012-1649.33.4.681

Main, M. \& Solomon, J. (1990). Procedures for identifying infants as disorganized/disoriented during the Ainsworth Strange Situation. En M. T. Greenberg, D. Cicchetti \& E. M. Cummings (Eds.), Attachment in the preschool years: Theory, research, and intervention (pp.121-160). Chicago, IL: University of Chicago Press.

Marrone, M. (2001). La teoría del apego: un enfoque actual. Madrid, España: Psimática. 
Mauricio, A. M. \& López, F. G. (2009). A latent classification of male batterers. Violence and Victims, 24, 419-438. https://doi.org/ $10.1891 / 0886-6708.24 .4 .419$

Melero, R. \& Cantero, M. J. (2008). Los estilos afectivos en la población española: un cuestionario de evaluación del apego adulto. Clínica y Salud, 19, 83-100.

Mikulincer, M., Shaver, P. R. \& Pereg, D. (2003). Attachment theory and affect regulation: The dynamics, development, and cognitive consequences of attachment-related strategies. Motivation and Emotion, 27, 77-102. https://doi.org/10.1023/A:1024515519160

Mora Ríos, J., Natera Rey, G., Tiburcio Sáinz, M. \& Juárez, F. (2008). Propiedades psicométricas de la Escala de Tácticas de Conflicto (CTS2) en mujeres mexicanas. Revista Mexicana de Psicología, 25, 107-117.

Moss, E., Parent, S., Gosselin, C., Rousseau, D. \& St-Laurent, D. (1996). Attachment and teacher-reported behavior problems during the preschool and early school-age period. Development and Psychopathology, 8, 511-525. https://doi.org/10.1017/S0954579400007240

O'Leary, K. D. \& Cascardi, M. (1998). Physical aggression in marriage: A developmental analysis. En T. N. Bradbury (Ed.), The developmental course of marital dysfunction (pp. 343-374). New York, NY: Cambridge University Press.

Organización Mundial de la Salud (2016). Violencia contra la mujer. Violencia de pareja y violencia sexual contra la mujer (Nota Descriptiva $\left.N^{\circ} 239\right)$. Genève, Suiza: Autor. Extraído de http://www.who.int/mediacentre/factsheets/fs239/es/

Organización Panamericana de la Salud (2002). Informe mundial sobre la violencia y la salud: resumen. Washington, DC: Autor. Extraído de http://www.who.int/violence_injury_prevention/violence/world_report/en/summary_es.pdf

Román, F., Rojas, G., Román, N., Iturry, M., Blanco, R., Leis, A. ... Grupo de Trabajo Programa Argencog (2012). Baremos del Test de la Mirada en español en adultos normales de Buenos Aires. Neuropsicología Latinoamericana, 4(3), 1-5.

San Miguel, M. T. (2006). Apego, trauma y violencia: comprendiendo las tendencias destructivas desde la perspectiva de la teoría del apego [Renn, P., 2006]. Aperturas Psicoanalíticas, 24, artículo 8. Extraído de http://www.aperturas.org/articulos.php?id $=0000423 \& a=$ Apego-trauma-y-violencia-comprendiendo-las-tendencias-destructivas-desde-la-perspectiva-de-la-teoria-del-apego\%5BRenn-P-2006\%5D

Servicio Nacional de la Mujer (2011). Plan nacional de acción para la prevención de la violencia intrafamiliar en Chile: septiembre 2011agosto 2012. Santiago, Chile: Autor, Unidad de Prevención en Violencia Intrafamiliar, Programa Chile Acoge Chile.

Shaver, P. R., Collins, N. \& Clark, C. L. (1996). Attachment styles and internal working models of self and relationship partners. En G. J. O. Fletcher \& J. Fitness (Eds.), Knowledge structures in close relationships: A social psychological approach (pp. 25-61). Mahwah, NJ: Lawrence Erlbaum.

Spencer, R., Guzmán, M., Fresno, A. \& Ramos, N. (2013). Validación chilena del cuestionario de evaluación del apego romántico Experiences in Close Relationships (ECR): análisis de la validez de criterio. Terapia Psicológica, 31, 313324. https://doi.org/10.4067/S0718-48082013000300006

Straus, M. A., Hamby, S. L., Boney-McCoy, S. \& Sugarman, D. B. (1996). The Revised Conflict Tactics Scales: Development and preliminary psychometric data. Journal of Family Issues, 17, 283-316. https://doi.org/.1177/019251396017003001

Taylor, G. J., Ryan, D. \& Bagby, R. M. (1985). Toward the development of a new self-report alexithymia scale. Psychotherapy and Psychosomatics, 44, 191-199. https://doi.org/10.1159/000287912

Walker, L. E. (1979). The battered women. New York, NY: Harper \& Row.

Waltz, J., Babcock, J. C., Jacobson, N. S. \& Gottman, J. M. (2000). Testing a typology of batterers. Journal of Consulting and Clinical Psychology, 68, 658-669. https://doi.org/101037/022-006X.68.4.658

Wei, M., Vogel, D. L., Ku, T. -Y. \& Zakalik, R. A. (2005). Adult attachment, affect regulation, negative mood, and interpersonal problems: The mediating roles of emotional reactivity and emotional cutoff. Journal of Counseling Psychology, 52, 14-24. https://doi.org/10.1037/0022-0167.52.1.14

Wimmer, H. \& Perner, J. (1983). Beliefs about beliefs: Representation and constraining function of wrong beliefs in young children's understanding of the deception. Cognition, 13, 103-128. https://doi.org/10.1016/0010-0277(83)90004-5

Fecha de recepción: Julio de 2015.

Fecha de aceptación: Marzo de 2017. 